\title{
Famílias e Consumo de Álcool em Adolescentes do Sexo Feminino: Uma Revisão Sistemática
}

\section{Families and Alcohol Consumption in Female Adolescents: A Systematic Review}

\author{
Ana Priscila de Oliveira Benites* \& Daniela Ribeiro Schneider \\ Universidade Federal de Santa Catarina, Florianópolis, Santa Catarina, Brasil
}

\begin{abstract}
Resumo
O consumo de álcool na adolescência é um fenômeno multideterminado, sendo que nele as relações familiares têm um papel destacado, tanto como fator protetor quanto como de risco. As adolescentes têm mudado seu padrão de consumo e aos poucos vem igualando ao dos meninos. Foi realizado levantamento bibliográfico na base Web of Science, entre 2006-2011, com os descritores girls, alcohol, family, com o objetivo de apresentar uma revisão sistemática do tema. Exibem-se dados bibliométricos, que indicam uma tendência de crescimento da produção científica nos últimos anos. Aprofundam-se os resultados em uma análise qualitativa, destacando-se o papel que a proximidade emocional com os pais e o monitoramento parental exercem enquanto fatores de proteção para as adolescentes. Palavras-chave: Álcool, adolescência, meninas, relações familiares, fatores de risco e proteção.
\end{abstract}

\begin{abstract}
Alcohol consumption by teenagers is a multi-determined phenomenon. Family relations play a prominent role in it and can be both a protection and a risk factor. Teenager girls have been changing their consumption pattern, becoming more and more similar to boys'. A bibliographic survey was carried out at Web of Science database, from 2006 to 2011, with the descriptors girls, alcohol and family, aiming to present a systematic review of the topic. Bibliometric data are shown, indicating a trend of growth in scientific production in the last years. The results are deepened through qualitative analyses of some articles. The role of emotional proximity with parents and parental monitoring is highlighted as protection factors for girls.

Keywords: Alcohol, adolescence, girls, family relations, risk and protective factors.
\end{abstract}

Há evidências de uma íntima relação entre adolescência e o início do uso de bebidas alcoólicas (Pratta \& Santos, 2006; Vieira, Ribeiro, \& Laranjeira, 2007). No "VI Levantamento Nacional sobre o Consumo de Drogas Psicotrópicas entre Estudantes do Ensino Fundamental e Médio das Redes Pública e Privada de Ensino nas 27 Capitais Brasileiras", realizado em 2010, o uso de álcool teve a menor média de idade (13 anos) dentre todas as drogas pesquisadas (Carlini et al., 2010).

Já o "I Levantamento Nacional sobre os padrões de consumo de álcool na população brasileira", realizado em 2007, encontrou uma alta frequência de adolescentes (9\%) que bebem mais do que uma vez por semana ( $12 \%$ meninos e $6 \%$ meninas). Embora os adolescentes também tenham apresentado alta taxa de abstinência, ocorre que aqueles que bebem têm a tendência de beber de uma forma problemática, como no caso do beber em "binge", no qual as taxas foram $21 \%$ dos meninos e $12 \%$ das meninas (Laran-

* Endereço para correspondência: Núcleo de Pesquisas em Psicologia Clínica (Psiclin). Departamento de Psicologia. Campus Universitário Reitor João David Ferreira Lima. Trindade - Florianópolis - Santa Catarina - Brasil. 88040-900. E-mail: anapriscila_benites@yahoo.com.br jeira, Pinsky, Zaleski, \& Caetano, 2007). Assim chamado pela literatura especializada, o padrão binge drinking, implica o consumo de cinco ou mais doses alcoólicas, por homens, ou de quatro ou mais doses, por mulheres, dentro do período de duas horas (National Institute on Alcohol Abuse and Alcoholism [NIAAA], s.d.).

Ainda que, legalmente, no Brasil, o álcool esteja proibido para consumo por menores de 18 anos (Estatuto da Criança e do Adolescente, 2005), seu poder de atração passa pelo fato de ser uma droga lícita, que, portanto, não tem a força de censura atribuída às substâncias ilegais. Os jovens vêem o álcool como um passaporte para a alegria, uma forma de relaxar e se divertir, que não é perigoso, sendo que muitos nem o consideram uma droga e muito menos as consequências do seu consumo (Custódio, 2009). Entretanto, pesquisas têm demonstrado que quanto mais precoce o contato com o álcool, mais o indivíduo se torna vulnerável à dependência (Pautassi, Camarini, Quadros, Miczek, \& Israel, 2010).

Apesar dos adolescentes serem encarados como grupo de risco para o uso de drogas, os fatores que podem levá-los a utilizá-las são variados e incluem a interação de múltiplos aspectos (Pratta \& Santos, 2006; Schenker, 2008). No caso, 
Schenker e Minayo (2005) consideram os relacionados ao indivíduo, família, grupo de pares, escola, comunidade e mídia, com destaque para as funções de risco e proteção que a família exerce no desenvolvimento.

Se, por um lado, o uso de bebida alcoólica em contextos familiares, religiosos e sociais, notadamente quando se convive com essas situações durante a infância, pode afetar tanto os padrões de consumo quanto a probabilidade de desenvolver problemas com o álcool (Associação Americana de Psiquiatria [APA], 2002), por outro, a qualidade da vida familiar, o papel dos pais na formação dos filhos e as relações familiares podem atuar como fatores protetores para o não envolvimento com drogas na adolescência (Pratta \& Santos, 2006). Algumas características familiares têm sido consideradas preditoras de uma conduta pró-social ou fator de proteção aos jovens. Entre elas, a existência de uma estrutura hierárquica definida na família, de uma boa qualidade da relação entre os pais, de um padrão de apego seguro pelo adolescente e de uma boa qualidade de comunicação do jovem com os pais e entre eles (Branco, Wagner, \& Demarchi, 2008).

No que diz respeito às particularidades de gênero, estudos têm comprovado que clima emocional familiar positivo age como fator protetor para o consumo de álcool e que esses mecanismos são diferentes para meninos e meninas (Kelly, O'Flaherty, et al., 2011). Contudo, ainda são escassas as referências que abordam os aspectos familiares direcionados às adolescentes usuárias de álcool e outras drogas (Guimarães, Hochgraf, Brasiliano, \& Ingberman, 2008).

O consumo de bebidas alcoólicas por adolescentes do sexo feminino tem merecido atenção pelas mudanças ocorridas nos últimos anos no seu padrão de uso. As pesquisas demonstram, inclusive, que elas já estão se aproximando em consumo aos seus pares do sexo oposto (Carlini et al., 2010). Assim, há que se pensar nas implicações desse comportamento para as adolescentes.

Por exemplo, estudo americano sobre o binge drinking apontou importante diferença dos efeitos do álcool no funcionamento cerebral de meninos e meninas, sendo que elas parecem ser mais vulneráveis aos efeitos neurotóxicos do uso pesado de álcool (Squeglia, Schweinsburg, Pulido, $\&$ Tapert, 2011). Além disso, após uma dose equivalente de bebida, a maior quantidade de gordura das mulheres leva a uma maior concentração de álcool no sangue, o que aumenta a potencialidade para a embriaguez (Edwards, Marshall, \& Cook, 2005).

Estudar como está ocorrendo o uso de álcool entre as adolescentes se faz necessário para o planejamento de programas específicos de promoção e prevenção em saúde, uma vez que o consumo de bebida alcoólica, neste grupo, tem sido relacionado à iniciação sexual precoce, múltiplos parceiros e à ocorrência da gravidez não planejada (O'Donnell et al., 2008; Pulcherio, Bastos, Strey, \& Boni, 2011). Deste modo, o objetivo do presente artigo foi realizar uma revisão sistemática das produções científicas internacionais envolvendo as mudanças no padrão do consumo de álcool por adolescentes do sexo feminino e seu contexto, destacando o papel de proteção e/ou risco das relações familiares.

\section{Método}

O estudo foi pautado em uma revisão sistemática da literatura especializada, tendo como foco pesquisas que abordassem a relação entre o consumo de álcool por adolescentes do sexo feminino e os diversos contextos familiares. A fonte consultada foi a base de dados Web of Science, de Thomson Reuters. Esta base é utilizada, habitualmente, em estudos que analisam a atividade científica porque acolhe todas as áreas científicas e tecnológicas e permite conhecer a repercussão ou o impacto das publicações a partir das citações recebidas pelos trabalhos publicados (Aleixandre-Benavent et al., 2009). O levantamento ocorreu em novembro de 2011, envolvendo os seguintes descritores no campo topic: girls, alcohol e family, com uma delimitação para os últimos seis anos, entre 2006 a 2011, realizando a intersecção entre os descritores com o algarismo booleano $A N D$ entre todas as equações de busca. Obteve-se, inicialmente, como resultado 208 artigos.

Procedeu-se, então, a uma análise de alguns indicadores bibliométricos, como o número de trabalhos publicados por ano, os países que mais produzem na temática, as principais instituições de pesquisa deste tema e as principais áreas em que os artigos foram publicados, buscando descrever o cenário internacional das pesquisas sobre o tema abordado.

Em seguida, alguns artigos foram selecionados para uma análise de conteúdo. Os critérios de inclusão utilizados foram: artigos completos ou de revisão (excluindo os resumos de comunicações em congressos, as correções, as reimpressões e as notícias), que abordavam especificamente a relação das famílias com o uso de álcool em adolescentes do sexo feminino, com ênfase em fatores de risco e proteção. Foram excluídos trabalhos: (a) que tivessem como foco outras drogas que não o álcool, no qual este aparecesse de forma secundária; (b) que tivessem como foco central fatores de risco para outros comportamentos que não o uso de álcool, como sexualidade, gravidez; (c) que centrassem sua análise exclusivamente em fatores genéticos, sem a inclusão de fatores psicossociais para o uso de álcool.

Passou-se, depois, a supervisão manual dos 208 artigos encontrados, atividade realizada por dois pesquisadores, sendo que estes chegaram a um consenso de 15 artigos completos que cumpriam todos os critérios elencados. Para a discussão qualitativa, os mesmos foram categorizados utilizando o método de Ruiz-Olabuénaga (2003), na qual parte-se de núcleos temáticos, analisando fenômenos que giram em torno dos focos de interesse da investigação. Realizou-se o processo de codificação dos dados, buscando encontrar as proximidades e similaridades aos diferentes textos, agrupando-os em categorias abrangentes, formando o que Ruiz-Olabuénaga (2003) chama de texto de investigação. Por fim, efetuou-se a verificação de tais categorias, 
Benites, A. P. O. \& Schneider, D. R. (2014). Famílias e Consumo de Álcool em Adolescentes do Sexo Feminino: Uma Revisão Sistemática.

no sentido de serem claras, significativas e exaustivas, resultando no texto apresentado. Os artigos foram agrupados em três núcleos temáticos para discussão: (a) relações familiares, gênero e consumo de álcool na adolescência, (b) eventos familiares e consumo de álcool na adolescência em meninos e meninas e (c) orientação parental na prevenção ao uso de álcool em meninas.

\section{Resultados}

Os resultados serão apresentados em dois blocos: o primeiro traz a discussão do cenário da produção científica internacional, incluídas em revistas de impacto, dos últimos seis anos (2006-2011) sobre o tema pesquisado. Em um segundo momento, realiza-se a análise de conteúdo de 15 artigos, escolhidos a partir dos critérios acima descritos, com o objetivo de aprofundar o tema e discutir os resultados das pesquisas mais atuais na temática abordada.

\section{Análise Bibliométrica}

A distribuição dos artigos referentes ao uso de álcool por meninas e sua relação com o ambiente familiar mostra uma tendência de crescimento da produção nos últimos cinco anos. $\mathrm{O}$ ano de 2007 teve um incremento na produção científica nesta temática, com 42 trabalhos publicados, representando um aumento em relação à produção do ano anterior, com 19 trabalhos. Em 2008, a produção teve uma leve queda, com 33 artigos, mantendo-se estável em 2009 e 2010, com 38 artigos em cada ano. Em 2011, quando a busca foi realizada, em novembro, já havia 39 artigos publicados, sem ainda ter se encerrado o ano.
O país com mais artigos publicados foi os Estados Unidos, com 105 trabalhos. Canadá foi o segundo, com 11 artigos, Brasil e Finlândia empatam em terceiro, com 10 trabalhos. Em seguida vem a França e a Suécia, com oito artigos cada e a Inglaterra e Holanda, com sete artigos. Colaborou na produção dos artigos o total de 52 países, sendo que dentre estes, somente cinco são da América Latina (Brasil, já citado; México, com seis artigos; Colômbia, com dois, Peru e Uruguai, com um trabalho cada).

A grande maioria dos trabalhos foi publicada em língua inglesa (195 artigos), sendo cinco trabalhos em português, quatro em espanhol e dois em francês. É importante ressaltar que a Web of Science é uma base de dados americana, que compila revistas de muitos países, mas a maioria das revistas indexadas é americana. Este pode ser um dado importante na análise do predomínio americano e da língua inglesa, ainda que, efetivamente, este país seja uma potência em produção científica (Vidal-Infer, 2010).

As instituições mais produtivas na temática são, também, americanas. A Univesity of Michigan foi a que teve mais artigos publicados nos seis anos analisados, chegando ao número de 10. Em seguida vem a Columbia University e a Iowa State University, com oito artigos cada.

Os artigos foram assinados pelo total de 938 autores, ainda que muitos se repitam por terem publicado mais de um artigo. Isto aponta uma média de 4,5 autores por artigo, indicando que a maioria dos artigos foi escrita em colaboração entre diferentes autores. Entre os autores mais produtivos, nesta temática, com mais de quatro artigos publicados nos anos analisados, estão seis americanos e um holandês, listados na Tabela 1.

Tabela 1

Autores Mais Produtivos sobre a Relação entre Uso de Álcool por Adolescentes e Família com Artigos Publicados na Web of Science, entre 2006 e 2011, com Instituição e País a que Pertencem (mais de quatro artigos)

\begin{tabular}{cccc}
\hline $\mathrm{N}^{\mathrm{o}}$ artigos & Autor & Instituição & País \\
\hline 6 & Fang, Lin & $\begin{array}{l}\text { University of Toronto } \\
\text { Columbia University }\end{array}$ & Estados Unidos \\
6 & Mason, W. Alex & $\begin{array}{c}\text { Boys Town National Research Inst. } \\
\text { for Child and Family Studies }\end{array}$ & Estados Unidos \\
6 & Schinke, Steven P. & Columbia University & Estados Unidos \\
6 & Spoth, Richard L. & Iowa State University & Estados Unidos \\
4 & Cole, Kristin C. & Columbia University & Estados Unidos \\
4 & Engels Rutger, C. M. E. & Maastricht University & Holanda \\
4 & Zucker, Robert A. & University of Michigan & Estados Unidos \\
\hline
\end{tabular}

Em termos das áreas de conhecimento que mais publicaram sobre a temática das meninas, uso de álcool e contextos familiares, a psicologia está em primeiro lugar, com 79 artigos, a área das drogadições em segundo, com 56 artigos e a área da saúde pública ocupacional, com 50 artigos. Com produção bem mais abaixo está a área da psiquiatria, com 33 artigos, a pediatria com 24 trabalhos e a Medicina Geral Interna, com 14. A Tabela 2 apresenta as áreas com mais produção sobre o tema. 
Tabela 2

Áreas de Conhecimento com Maior Produção sobre a Relação entre Uso de Álcool por Adolescentes e Família Publicados na Web of Science entre 2006 e 2011 (mais de cinco trabalhos)

\begin{tabular}{lc}
\hline \multicolumn{1}{c}{ Áreas do Conhecimento } & $\mathrm{N}^{\mathrm{o}}$ artigos \\
\hline Psychology & 79 \\
Substance Abuse & 56 \\
Public Environmental Occupational Health & 50 \\
Psychiatry & 33 \\
Pediatrics & 24 \\
General Internal Medicine & 14 \\
Family Studies & 8 \\
Neurosciences Neurology & 8 \\
Social Work & 7 \\
Criminology Penology & 6 \\
\hline
\end{tabular}

Tabela 3

Lista das Revistas com Maior Produção sobre a Relação entre Uso de Álcool por Adolescentes e Família Publicados na Web of Science entre 2006 e 2011 (mais de três artigos)

\begin{tabular}{lc}
\hline \multicolumn{1}{c}{ Revistas } & $\mathrm{N}^{\mathrm{o}}$ artigos \\
\hline BMC Public Health & 11 \\
Journal of Adolescent Health & 9 \\
$\begin{array}{l}\text { Alcoholism Clinical and Experimental } \\
\text { Research }\end{array}$ & 8 \\
Journal of Youth and Adolescence & 8 \\
Drug and Alcohol Dependence & 7 \\
Addictive Behaviors & 6 \\
Journal of Studies on Alcohol and Drugs & 6 \\
Psychology of Addictive Behaviors & 5 \\
Substance Use Misuse & 5 \\
Addiction & 4 \\
Archives of Pediatrics Adolescent Medicine & 4 \\
Cadernos de Saúde Pública & 3 \\
Journal of Community Psychology & 3 \\
Salud Mental & 3 \\
\hline
\end{tabular}

A revista que mais publicou artigos sobre o tema estudado foi a BMC Public Health, com 11 trabalhos, seguida pela revista Journal of Adolescent Health, com nove trabalhos. Em terceiro lugar estão as revistas Alcoholism Clinical and Experimental Research e Journal of Youth and Adolescence, com oito artigos. A primeira revista brasileira que aparece é a Cadernos de Saúde Pública, com três artigos. Na Tabela 3 são apresentadas as revistas com maior número de artigos na temática em pauta.

Verifica-se que a produção científica na temática de adolescentes do sexo feminino, uso de álcool e relações familiares, conforme aparece na base de dados da $\mathrm{Web}$ of Science, teve uma tendência de aumento gradual entre os anos de 2006 e 2011, sendo a maior parte dos estudos desenvolvida por universidades e pesquisadores americanos, cuja produção é prioritariamente em língua inglesa. Dos países da América Latina, o Brasil foi o primeiro em produção, ainda que muito aquém da produção americana. A maioria dos trabalhos foi realizada em colaboração entre diferentes pesquisadores e instituições. As áreas da psicologia e de drogadição foram as que mais publicaram artigos sobre o tema.

\section{Análise dos Artigos Selecionados}

Relações Familiares, Gênero e Consumo de Álcool na Adolescência. Nesta temática reuniram-se pesquisas que abordassem as relações familiares e sua influência no consumo de álcool em meninos e meninas.

Estudo realizado com 1.028 adolescentes finlandeses e suas famílias, acompanhados por 15 anos, não encontrou diferenças significativas no uso de álcool entre adolescentes do sexo feminino e masculino, de maneira que as análises foram feitas conjuntamente. O principal resultado da pesquisa trouxe como fatores mais potentes para o uso problemático de álcool aos 15 anos, o uso pesado de bebida alcoólica pelo pai e a experiência precoce do adolescente com o álcool (Seljamo, Aromaa, Koivusilta, Rautava, \& Sourander, 2006).

As características familiares exercem influências distintas em adolescentes do sexo feminino e masculino; partindo dessa premissa, foi realizada uma pesquisa com 6.837 adolescentes australianos, na faixa etária de 11 a 13 anos, com o objetivo de examinar as diferenças de gênero em associação com o comportamento parental em relação ao uso de álcool e a qualidade das relações familiares. Os resultados indicaram a proximidade emocional com o genitor do sexo oposto como um fator protetor para ambos os sexos, ao passo que conflitos familiares estiveram associados com o consumo de bebida alcoólica em meninas. Os autores discutem que essa proximidade emocional com o genitor do sexo oposto parece ser especialmente importante nos períodos de transição, como a entrada no ensino médio e o início da puberdade. Já em relação ao uso de álcool e conflitos familiares, argumentam que outros estudos, também, sugerem uma maior vulnerabilidade das meninas a esses conflitos e indicam a necessidade de se considerar as especificidades de gênero para se pensar estratégias de prevenção orientadas para a família (Kelly, Toumbourou, et al., 2011). 
Outro estudo realizado com 855 estudantes australianos, no início e no meio da adolescência, investigou as diferenças de gênero e o impacto do clima emocional familiar no uso do álcool pelo adolescente com sua exposição a grupos de pares que fazem uso de bebida alcoólica. Nesta pesquisa, a proximidade com a mãe esteve associada a uso menos frequente de álcool em meninas, efeito que também reduziu o envolvimento delas com pares desviantes. Quanto à influência dos grupos de pares, os autores discutem que a cultura do uso do álcool pelos meninos faz com que eles exerçam mais influência nas meninas, do que o contrário no que diz respeito ao comportamento de beber - o que as tornam mais vulneráveis à socialização com o álcool pelos meninos. Enfim, o estudo destaca a importância da proximidade emocional entre mãe e filha na prevenção ao uso de álcool (Kelly, O'Flaherty, et al., 2011).

Também interessado nas diferenças de gênero na dinâmica familiar, a pesquisa de Okulicz-Kozaryn (2010) teve como objetivo investigar como as mudanças na estrutura familiar (famílias patriarcais x famílias igualitárias) têm influenciado o consumo de álcool em adolescentes do sexo feminino. Embasada na teoria do controle social, o estudo foi realizado com 3.087 adolescentes poloneses, com média de idade de 14,5 anos. Os resultados mostraram que meninas de famílias patriarcais que passam mais tempo com suas mães e percebem maior controle materno têm menos riscos de se embriagarem. Deste modo, os autores sugerem que, especialmente, as mães de famílias igualitárias, nas quais homem e mulher desempenham funções semelhantes, supervisionem atentamente o comportamento das filhas e passem mais tempo com elas.

Estágios do consumo de álcool na adolescência podem ser diferentemente afetados pelo uso de álcool pelos familiares e amigos. Nesse sentido, estudo longitudinal realizado nos Países Baixos com 3.760 gêmeos, com média de idade de 17,8 anos, investigou a associação entre o uso de álcool pelos adolescentes com o consumo de álcool por parte de mães, pais, irmãos e amigos (Scholte, Poelen, Willemsen, Boomsma, \& Engels, 2008).

A pesquisa apontou risco maior para o uso regular de álcool em adolescentes de 12-15 anos, cujos irmãos gêmeos do mesmo sexo e amigos faziam uso regular de bebida alcoólica - risco que tendeu a diminuir com a idade. Os resultados foram similares para meninos e meninas, a única diferença foi entre irmãs gêmeas uni vitelinas, que mostraram risco maior para consumo de álcool na entrada da vida adulta, quando uma das irmãs fazia uso. Os autores atribuíram o fenômeno, ao fato de irmãs gêmeas interagirem mais entre si do que irmãos gêmeos na vida adulta (21-25 anos), o que resultaria em uma maior similaridade no padrão de consumo de álcool entre elas (Scholte et al., 2008).

No estudo de Yeh, Chiang e Huang (2006), realizado com 771 adolescentes taiwaneses, o consumo de álcool pelo pai exerceu influência no uso de álcool pelos filhos, ao passo que consumo de álcool pela mãe influenciou o uso nas filhas. Meninas que bebiam álcool mostraram-se mais sensíveis às normas e relações com os pares e foram, significativamente, influenciadas pela atitude de aprovação do grupo quanto ao comportamento de beber. Também para elas o uso de bebida esteve mais estreitamente associado à dinâmica familiar.

Eventos Familiares e o Consumo de Álcool na Adolescência em Meninos e Meninas. Aqui se agruparam pesquisas que associassem alguns eventos familiares com o consumo de álcool pelos adolescentes, tais como episódios estressantes da infância e adolescência e/ou situações do cotidiano familiar. O estudo de Burk et al. (2011) focou como as diferenças de gênero em conjunto com fatores distais da infância e proximais da adolescência influenciam no consumo de álcool na adolescência. Fundamentada na teoria Epigenética, a pesquisa contou com 362 adolescentes americanos, com média de idade de 16 anos, que foram acompanhados desde a gestação materna. Os resultados mostraram que maior uso de álcool pelos pais esteve relacionado ao aumento do consumo em meninos e meninas, sendo esse efeito mais forte nas meninas. Ainda, comportamento desinibido precoce na infância em interação com alto estresse familiar previu maior uso de álcool nas adolescentes. Os autores discutem o risco para o consumo de álcool em meninas como consequência de efeitos independentes de riscos individuais e contextuais da infância, articulados ao risco social da adolescência, sugerindo que estratégias de prevenção para o uso de álcool em meninas devam ser pensadas antes dessa fase.

Embasada na Teoria Bioecológica de Bronfenbrenner (Bronfenbrenner, 2011), pesquisa finlandesa investigou o significado dos eventos familiares da adolescência e sua relação com depressão e episódios de embriaguez em 3.242 adolescentes. Eventos como desemprego parental, envolvimento dos pais com a justiça e dificuldades financeiras estiveram associados com embriaguez nos adolescentes. Todavia, a associação entre determinados acontecimentos familiares e uso frequente de álcool não pode ser vista como independente de outros eventos, por isso os autores argumentam que um modelo de estresse cumulativo pode explicar a associação entre eventos familiares e o consumo de bebida alcoólica. Em relação às diferenças de gênero, vários eventos familiares estiveram associados à embriaguez nas meninas, ao passo que para os meninos sobressaíram-se as discussões entre os pais e as dificuldades financeiras na família. Estudos sobre o consumo de álcool na vida adulta sugerem que eventos estressantes estão mais relacionados ao beber masculino do que feminino - o resultado dessa pesquisa, contudo, implica que a situação pode ser diferente no meio da adolescência. Ainda, os meninos reagiram ao estresse familiar com mais sintomas internos (depressão), ao passo que as meninas apresentaram tanto sintomas internos, quanto externos (embriaguez); Frojd, Kaltiala-Heino, Pelkonen, Von Der Pahlen, \& Marttunenf, 2009).

Uma pesquisa realizada, na Lituânia, com 369 estudantes da $5^{\mathrm{a}}$ série sobre o tempo que a família passa junto 
versus uso do cigarro e álcool, trouxe que meninos cujos pais frequentavam festas com álcool, faziam mais uso de bebida alcoólica. Quanto à atividade familiar conjunta mais frequente, para as meninas foi comer junto uma refeição, já para os meninos foi realizar atividades esportivas com os pais. No entanto, para elas, esse tempo junto com a família apareceu como um fator protetor para evitar o uso de álcool (Garmiene, Zemaitiene, \& Zaborskis, 2006). Resultado similar ao encontrado em um estudo longitudinal com 5.511 adolescentes americanos (3.283 meninos e 2.228 meninas), no qual jantar diariamente com a família também foi protetivo para as meninas (Fisher, Miles, Austin, Camargo, \& Colditz, 2007).

Orientação Parental na Prevenção ao Uso de Álcool em Meninas. Nesta temática foram agrupados quatro artigos americanos e um francês. A pesquisa de Schinke, Fang e Cole (2008) foi realizada com 781 adolescentes do sexo feminino com média de idade de 12,6 anos, sendo que $40,6 \%$ delas já haviam feito uso de álcool, e suas mães. O recrutamento da amostra foi feito por meio de anúncios espalhados na cidade de Nova Iorque para responder a um questionário on-line. Os autores discutem que o paradeiro das adolescentes, depois da aula, o uso de drogas por colegas e outras variáveis psicossociais têm sido utilizadas para explicar o risco do uso de drogas na adolescência, contudo, pouca atenção tem sido dada para a influência do comportamento, monitoramento e normas parentais. Neste estudo, mães que faziam uso de bebida alcoólica estiveram relacionadas ao uso de álcool nas filhas. Por outro lado, mães que sabiam o paradeiro das filhas foi associado a reduzido risco de uso de álcool nas adolescentes. De maneira geral, nas famílias nas quais há regras sobre o não uso de drogas é mais provável que as filhas as incorporem, para reduzirem seu comportamento de risco.

Em outro artigo, os mesmos autores investigam como fatores demográficos, psicológicos, relacionados aos grupos de pares e à família poderiam explicar o uso de álcool em adolescentes do sexo feminino. A amostra contou com 1.187 adolescentes de 10 a 14 anos e suas mães - também recrutadas via anúncios espalhados na cidade de Nova Iorque para responderem a um questionário on-line - e trouxe como resultados que $39,7 \%$ das adolescentes já haviam feito uso de álcool na vida. Ser mais velha, ter baixo rendimento escolar, maiores níveis de depressão, maior percepção de uso de álcool entre os colegas e alto nível de uso de álcool pela mãe foram fatores observados no grupo que já fez uso de álcool e que bebeu no último mês. Por outro lado, alto nível de autoestima com o corpo, auto-eficácia, monitoramento parental, regras familiares contra o uso de álcool e envolvimento familiar foram fatores encontrados no grupo de adolescentes que não fazia uso de álcool. Os autores salientam, contudo, as limitações do estudo, dentre elas o fato dos dados terem sido coletados, exclusivamente, via Internet (Fang, Schinke, \& Cole, 2009).

Outra pesquisa americana realizada com meninas de $6^{\mathrm{a}}$ série e seus pais investigou as práticas parentais relacio- nadas aos riscos das filhas para uso de álcool e comportamentos sexuais. Foram entrevistadas 771 adolescentes com média de idade de 12 anos e seus pais, recrutados em sete escolas de áreas de baixa renda da cidade de Nova Iorque. Apesar de apenas quatro pais terem relatado que sabiam que suas filhas já haviam consumido álcool, 22\% delas beberam álcool no último ano. De modo que os pais, substancialmente, subestimaram os comportamentos de riscos das suas filhas relacionados ao envolvimento com rapazes e ao uso de álcool. Todavia, meninas que relataram ter supervisão parental, regras e desaprovação dos pais para se engajarem em comportamentos de risco apresentaram menos propensão a fazerem uso de álcool (O’Donnell et al., 2008). Em uma pesquisa com 16.532 adolescentes franceses, o controle e o suporte emocional parental, também, apareceram como fatores protetores para o não uso de álcool pelas meninas (Choquet, Hassler, Morin, Falissard, \& Chau, 2008).

Por fim, o estudo de Costello, Sung, Worthmann e Angold (2007) investigou a entrada na puberdade e sua relação com o início do uso de álcool em 1.420 adolescentes americanos, com idades entre 9 e 13 anos. Diversos fatores foram pesquisados, dentre eles a relação da puberdade precoce com aspectos do contexto familiar. Nesse quesito, o resultado apontou que supervisão familiar inconsistente aumenta o risco do uso do álcool em meninas que entram precocemente na puberdade.

\section{Considerações Finais}

A análise realizada aponta o incremento das pesquisas e colaborações científicas nos últimos anos sobre o tema do consumo de álcool e outras drogas por adolescentes do sexo feminino, em especial relacionadas aos fatores de risco e proteção que o contexto familiar pode representar, na medida em que o padrão de consumo das meninas vem aumentando e, pouco a pouco, se igualando ao padrão dos meninos, demonstrando a preocupação da comunidade científica com o assunto.

Países latino-americanos têm pouca inserção de suas produções nas bases de dados com fator de impacto internacional, com menos de $10 \%$ dos artigos publicados no período, com uma clara hegemonia norte-americana na área. Estes dados indicam que os pesquisadores deste continente necessitam incrementar mais pesquisas e publicações com fator de impacto internacional, na medida em que esta é uma problemática bastante presente na realidade sociocultural destes países e sua contribuição científica se faz importante na sua elucidação.

$\mathrm{Na}$ análise de conteúdo, as pesquisas apresentadas, ainda que sejam de países diversos, trazem alguns pontos em comum, tais como a importância da proximidade emocional entre mãe e filha para o não uso de álcool (Kelly, O'Flaherty, et al., 2011; Okulicz-Kozaryn, 2010) e a influência do consumo de álcool materno no consumo das filhas (Fang et al., 2009; Schinke et al., 2008; Yeh et al., 2006). 
Benites, A. P. O. \& Schneider, D. R. (2014). Famílias e Consumo de Álcool em Adolescentes do Sexo Feminino: Uma Revisão Sistemática.

Os estudos destacam que os fatores implicados no uso de álcool em meninos e meninas têm particularidades distintas, tanto no que diz respeito aos aspectos biológicos, quando aos sociofamiliares, indicando a necessidade de aprofundar as pesquisas sobre tais contextos específicos para os gêneros.

Em relação à figura paterna, o uso de álcool por parte de pai e mãe (Burk et al., 2011) exerceu influência no consumo dos filhos (ambos os sexos), já na pesquisa de Seljamo et al. (2006), o consumo paterno esteve associado principalmente ao consumo das filhas. Vale ressaltar que no estudo de Kelly, Toumbourou, et al. (2011), a proximidade emocional com o genitor sexo oposto apareceu como fator protetor para as meninas nos períodos de transição, tais como o início da puberdade e a entrada no ensino médio.

Eventos estressantes na adolescência e conflitos familiares também estiveram associados ao uso de álcool em meninas (Frojd et al., 2009; Kelly, Toumbourou, et al., 2011), ao passo que regras claras sobre o não uso de álcool e monitoramento parental atuaram como fatores protetores (Choquet et al., 2008; Costello et al., 2007; Fang et al., 2009; O’Donnell et al., 2008; Schinke et al., 2008). Nessa perspectiva, O'Donnell et al. (2008) destacam a importância dos limites e da supervisão parental para as meninas. No estudo de Kelly, Toumbourou, et al. (2011), a desaprovação parental do consumo de álcool atuou como um significativo fator protetor a par de outras variáveis.

No que diz respeito ao subsistema fraternal, a pesquisa de Scholte et al. (2008) trouxe risco maior para o uso de álcool entre irmãos gêmeos do mesmo sexo, o que indica a proximidade de gênero entre os membros da família como um fator importante de influência para o uso de álcool, tal quando mães e filhas bebem. Por fim, partilhar as refeições em família tem um efeito protetor para as adolescentes em relação ao consumo de álcool (Fisher et al., 2007; Garmiene et al., 2006).

Boa parte das famílias não se dá conta da grandeza da sua tarefa, - que é a de formar novos cidadãos, trabalho no qual, necessariamente, estão imbricados valores familiares e socioculturais (Schenker, 2008). Esses valores são importantes fatores no processo de socialização de crianças e adolescentes, do qual o álcool, muitas vezes, acaba fazendo parte. Por isso, dada as especificidades desse processo para meninos e meninas, as relações do contexto familiar precisam ser levadas em consideração quando se pensa sobre o consumo de álcool em adolescentes do sexo feminino, no sentido de se formular estratégias de prevenção voltadas para esse grupo, o que indica a necessidade de mais pesquisas voltadas a essa temática.

\section{Referências}

Aleixandre-Benavent, R., Alonso, A., Chorro, F. G., Alfonso, F. M., Gozález, G. A., Salvador, M. J. T., ...Martin-Burrieza, F. (2009). La producción científica cardiovascular en España y en el contexto europeo y mundial (2003-2007). Revista Española de Cardiologia, 62(12), 1404-1417.
Associação Americana de Psiquiatria. (2002). DSM-IV-TR, Manual Diagnóstico e Estatístico de Transtornos Mentais (4. ed. rev.). Porto Alegre, RS: Artmed.

Branco, B. M., Wagner, A., \& Demarchi, K. A. (2008). Adolescentes infratores: Rede social e funcionamento familiar. Psicologia Reflexão e Crítica, 21(1), 125-132.

Bronfenbrenner, U. (2011). Bioecologia do desenvolvimento humano: Tornando os seres humanos mais humanos. Porto Alegre, RS: Artmed.

Burk, L. R., Armstrong, J. M., Goldsmith, H. H., Klein, M. H., Strauman, C. P., \& Essex, M. J. (2011). Sex, temperament, and family context: How the interaction of early factors differentially predict adolescent alcohol use and are mediated by proximal adolescent factors. American Psychological Association, 25(1), 1-15.

Carlini, E. L. A., Noto, A. R., Sanchez, Z. V. D. M., Carlini, C. M. A., Locatelli, D. P., Abeid, L. R., et al.(2010). VI Levantamento Nacional sobre o Consumo de Drogas Psicotrópicas entre Estudantes do Ensino Fundamental e Médio das Redes Pública e Privada de Ensino nas 27 Capitais Brasileiras -2010. São Paulo: CEBRID - Centro Brasileiro de Informações sobre Drogas Psicotrópicas: UNIFESP - Universidade Federal de São Paulo. Brasília: SENAD - Secretaria Nacional de Políticas sobre Drogas.

Choquet, M., Hassler, C., Morin, D., Falissard, B., \& Chau, N. (2008). Perceived parenting styles and tobacco, alcohol and cannabis use among French adolescents: Gender and family structure differentials. Alcohol and Alcoholism, 43(1), 73-80.

Costello, E. J., Sung, M., Worthman, C., \& Angold, A. (2007). Pubertal maturation and the development of alcohol use and abuse. Drug and Alcohol Dependence, 88(Suppl. 1), S50-S59.

Custódio, D. K. A. S. (2009). Álcool e sociabilidade: A farra das adolescentes (Dissertação de mestrado, Universidade Federal do Rio Grande do Norte, Natal, RN, Brasil).

Edwards, G., Marshall, E. J., \& Cook, C. C. H. (2005). O tratamento do alcoolismo: Um guia para profissionais da saúde (4. ed.). Porto Alegre, RS: Artmed.

Estatuto da Criança e do Adolescente. (2005). Lei $N^{o}$ 8.069, de 13 de Julho de 1990. Dispõe sobre o Estatuto da Criança e do Adolescente e dá outras providencias. Brasília, DF: Ministério da Educação.

Fang, L., Schinke, S. P., \& Cole, K. C. (2009). Underage drinking among young adolescent girls: The role of family processes. American Psychological Association, 23(4), 708-714.

Fisher, L. B., Miles, I. W., Austin, B., Camargo, C. A., Jr., \& Colditz, G. A. (2007). Predictors of initiation of alcohol use among US adolescents: Findings from a prospective cohort study. Archives of Pediatrics \& Adolescent Medicine, 161(10), 959-966.

Frojd, S., Kaltiala-Heino, R., Pelkonen, M., Von Der Pahlen, B., \& Marttunenf, M. (2009). Significance of family life events in middle adolescence: A survey on Finnish community adolescents. Nordic Journal of Psychiatry, 63(1), 78-86.

Garmiene, A., Zemaitiene, N., \& Zaborskis, A. (2006). Family time, parental behaviour model and the initiation of smoking and alcohol use by teen-year-old children: An epidemiological study in Kaunas, Lithuania. BMC Public Health, 6, 287.

Guimarães, A. B. P., Hochgraf, P. B., Brasiliano, S., \& Ingberman, Y. K. (2008). Aspectos familiares de meninas adolescentes dependentes de álcool e drogas. Revista de Psiquiatria Clínica, 36(2), 69-74. 
Kelly, A. B., O'Flaherty, M., Toumbourou, J. W., Connor, J. P., Hemphill S. A., \& Catalano, R. F. (2011). Gender differences in the impact of families on alcohol use: A lagged longitudinal study of early adolescents. Addiction, 106(8), 1427-36.

Kelly, A. B., Toumbourou, J. W., O'Flaherty, M., Patton, G. C., Homel, R., Connor, J. P., \& Williams, J. (2011). Family relationship quality and early alcohol use: Evidence for gender-specific risk processes. Journal of Studies on Alcohol and Drugs, 72, 399-407.

Laranjeira, R., Pinsky, I., Zaleski, M., \& Caetano, R. (2007). I Levantamento Nacional sobre os padrões de consumo de álcool na população brasileira. Brasília, DF: Secretaria Nacional Antidrogas.

Moreno, R. S., Ventura, R. N., \& Brêtas, J. R. S. (2010). O uso de álcool e tabaco por adolescentes do município de Embu, São Paulo, Brasil. Revista da Escola de Enfermagem da USP, 44(4), 969-977.

National Institute on Alcohol Abuse and Alcoholism. (s.d.). Moderate \& Binge Drinking. Retrieved June 24, 2012, from http://www.niaaa.nih.gov/alcohol-health/overview-alcoholconsumption/moderate-binge-drinking

O’Donnell, L., Stueve, A., Duran, R., Myint-U, A., Agronick, G., Doval, A. S., \& Wilson-Simmons, R. (2008). Parenting practices, parents' underestimation of daughters' risks, and alcohol and sexual behaviors of urban girls. Journal of Adolescent Health, 42(5), 496-502.

Okulicz-Kozaryn, K. (2010). Gender and family differences in adolescent's heavy alcohol use: The power-control theory perspective. Health Education Research, 25(5), 780-791.

Pautassi, R. M., Camarini, R., Quadros, I. M., Miczek, K. A., \& Israel, Y. (2010). Genetic and environmental influences on ethanol consumption: Perspectives from preclinical research. Alcohol Clinical and Experimental Research, 34(6), 976-987.

Pratta, E. M. M., \& Santos, M. A. (2006). Reflexões sobre as relações entre drogadição, adolescência e família: Um estudo bibliográfico. Estudos de Psicologia (Natal), 11(3), 315-322.

Pulcherio, G., Bastos, T., Strey, M., \& Boni, R. (2011). Consumo de álcool entre adolescentes do sexo feminino. Revista de Psiquiatria Clínica, 38(5), 209-210.

Ruiz-Olabuénaga, J. I. (2003). Metodología de la investigación cualitativa. Bilbao, España: Universidad de Deusto. Retrieved June 20, 2012, from http://www.fileden.com/ files/2011/4/1/3107708/metodologia_de_la_investigacion_ cualitativa\%20olagabuena.pdf

Schenker, M. (2008). Valores familiares e uso abusivo de drogas. Rio de Janeiro, RJ: Fundação Oswaldo Cruz.

Schenker, M., \& Minayo, M. C. S. (2005). Fatores de risco e proteção para o uso de drogas na adolescência. Ciência \& Saúde Coletiva, 10(3), 707-717.

Schinke, S. P., Fang, L., \& Cole, K. C. A. (2008). Substance use among early adolescent girls: Risk and protective factors. Journal of Adolescent Health, 43, 191-194.

Scholte, R. H. J., Poelen, E. A. P, Willemsen, G., Boomsma, D. I., \& Engels, R. C. M. E. (2008). Relative risks of adolescent and young adult alcohol use: The role of drinking fathers, mothers, siblings and friends. Addictive Behaviors, 33, 1-14.

Seljamo, S., Aromaa, M., Koivusilta, L., Rautava, P., \& Sourander, A. (2006). Alcohol use in families: A 15-year prospective follow-up study. Addiction, 101, 984-992.

Squeglia, L. M., Schweinsburg, A. D., Pulido, C., \& Tapert, S. F. (2011). Adolescent binge drinking linked to abnormal spatial working memory brain activation: Differential gender effects. Alcohol Clinical and Experimental Research, 35(10), 1831-1841.
Strauch, E. S., Pinheiro, R. T., Silva, R. A., \& Horta, B. L. (2009). Uso de álcool por adolescentes: Estudo de base populacional. Revista de Saúde Pública, 43(4), 647-55.

Vidal-Infer, A. M. (2010). Análisis de los artículos originales publicados en revistas especificas sobre drogodependencias incluidas en el Journal Citation Reports (2002-2006) (Unpublished doctoral dissertation, Universidad de Valencia, Valencia, Spain).

Vieira, D. L., Ribeiro, M., \& Laranjeira, R. (2007). Evidence of association between early alcohol use and risk of later problems. Revista Brasileira de Psiquiatria, 29(3), 222-227.

Vieira, D. L., Ribeiro, M., Romano, M., \& Laranjeira, R. R. (2007). Álcool e adolescentes: Estudo para implementar políticas municipais. Revista de Saúde Pública, 41(3), 396-403.

Yeh, M., Chiang, I., \& Huang, S. (2006). Gender differences in predictors of drinking behavior in adolescents. Addictive Behaviors, 31, 1929-1938. 